\title{
METU-DEFOCUSING BEAMLINE : A 15- 30 MeV PROTON IRRADIATION FACILITY and BEAM MEASUREMENT SYSTEM
}

\author{
M.Bilge Demirkoz \\ Middle East Technical University \\ Ankara, Turkey \\ demirkoz@metu.edu.tr \\ Besna Bulbul \\ Middle East Technical University \\ Ankara, Turkey \\ besna.bulbul@metu.edu.tr \\ Yusuf Orhan \\ Ministry of Defense \\ Ankara, Turkey \\ yusuf.orhan@msb.gov.tr \\ Caglar Saral \\ Middle East Technical University \\ Ankara, Turkey \\ csaral@metu.edu.tr \\ Ilias Efthymiopoulos \\ CERN \\ Geneva, Switzerland \\ ilias.efthymiopoulos@cern.ch \\ Abdulrahman Albarodi \\ Middle East Technical University \\ Ankara, Turkey \\ abdul.albaroudi@metu.edu.tr
}

\author{
Caner Seckin \\ Middle East Technical University \\ Ankara, Turkey \\ cseckin@metu.edu.tr \\ Pelin Uslu \\ Middle East Technical University \\ Ankara, Turkey \\ puslu@metu.edu.tr \\ Selen Akcelik \\ Middle East Technical University \\ Ankara, Turkey \\ sakcelik@metu.edu.tr \\ Selcen Uzun Duran \\ Karadeniz Technical University \\ Trabzon, Turkey \\ selcenduran@ktu.edu.tr \\ Anıl Berkay Poyrazoglu \\ Middle East Technical University \\ Ankara, Turkey \\ apoyraz@metu.edu.tr \\ Nazire Celik \\ Middle East Technical University \\ Ankara, Turkey \\ nazcelik@metu.edu.tr
}

\author{
Akanay Avaroglu \\ Middle East Technical University \\ Ankara, Turkey \\ akanay@metu.edu.tr \\ Erinc Kilic \\ Middle East Technical University \\ Ankara, Turkey \\ erinck@metu.edu.tr \\ Merve Yigitoglu \\ Middle East Technical University \\ Ankara, Turkey \\ merve.yigitoglu@metu.edu.tr \\ Ugur Kilic \\ Middle East Technical University \\ Ankara, Turkey \\ ukilic@metu.edu.tr
}

\begin{abstract}
Middle East Technical University - Defocusing Beam Line (METU-DBL) project is an irradiation facility providing $15 \mathrm{MeV}$ to $30 \mathrm{MeV}$ kinetic energy protons for testing various high radiation level applications, ranging from Hi-Lumi LHC upgrade, space electronic components to nuclear material research. The project located inside the premises of the TAEA (Turkish Atomic Energy Agency) SANAEM (Saraykoy Nuclear Education and Research Center) close to Ankara, provides users a wide selectable flux menu $\left(10^{5}-10^{10} \mathrm{p} / \mathrm{cm}^{2} / \mathrm{s}\right)$. The facility is now being commissioned and the facility will be providing a large test area $(20 \mathrm{~cm} \times 15 \mathrm{~cm})$ for material, detector and electronics tests. The proton beam is monitored along the beamline using
\end{abstract}

Keywords-Single Event Effect, Defocusing beamline, Space Radiation, Proton Accelerator Facility, Radiation tests

\section{INTRODUCTION}

Electronic devices are exposed to high dose radiation in the Hi-Lumi $\mathrm{LHC}$ [1] and space environment. Radiation exposure to these devices affects their performance and the reliability so they must be tested. In the scope of METU-DBL (Defocusing Beam Line) project, a beamline has been constructed at Turkish Atomic Energy Authority (TAEA)'s proton accelerator facility's (PAF) R\&D room. The schematic design of the proton accelerator facility is given in Fig. 1, where a central cyclotron provides proton beams to two simultaneous irradiation rooms. METU-DBL's goal is to study single event effects (SEEs), are aluminum oxide screens and the flux and uniformity is measured using three detectors attached to the robotic system for cross- checks. A fiber scintillator detector scans the large irradiation area while small area diamond detector and Timepix 3 detector are used for spot checks for calibration. Several samples can be radiated simultaneously inside the irradiation area and the robotic system provides 5 separate holders for samples which can be moved in or out, providing users flexibility for the desired fluence. This talk will first introduce METU- DBL as a radiation test facility, then discuss the radiation monitoring of the beam area and the radiation room, while highlighting how this facility can be used for future testing of materials for radiation tolerance.

caused by single, energetic particles, namely protons. In the space environment, protons which are trapped in radiation belts around the Earth, or solar flares, can cause both direct ionization and indirect ionization SEEs in sensitive electronic devices [2].

TAEA SANAEM PAF has a selectable energy range 15$30 \mathrm{MeV}$ cyclotron proton accelerator in the range of $0.1 \mu \mathrm{A}$ to $1.2 \mathrm{~mA}$. This facility has four irradiation rooms. Three of them are used in order to produce radiopharmaceuticals for medical purposes and the other room is reserved for R\&D purposes [3]. 


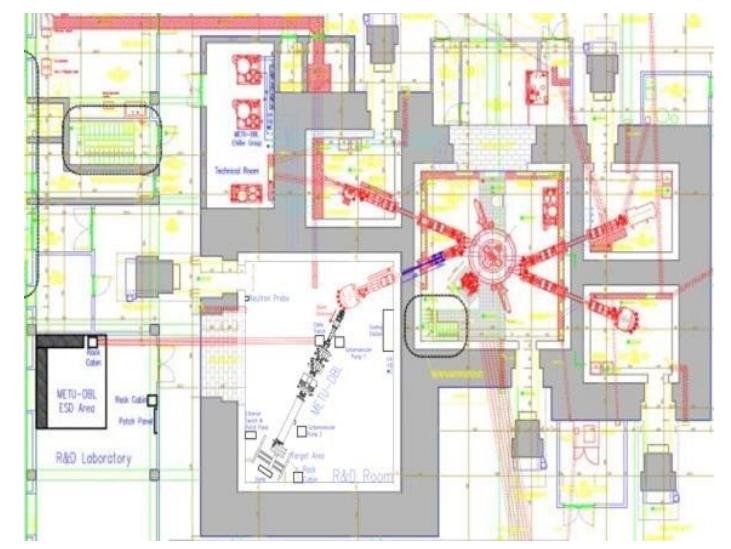

Fig. 1. The schematic design of TAEA SANAEM Proton Accelerator Facility. The cyclotron is seen in red on the right, where 4 arms extend out to 4 different irradiation rooms. The one on the lower left is the R\&D room in which METU-DBL is shown in black. The connection between this R\&D room and the control system in the R\&D laboratory is provided by the underground trench shown here in red.

Proton tests are crucial for space applications because protons are the dominant component of cosmic rays that penetrate through the shielding of a satellite. For radiation tests, METU-DBL follows the ESA/ESCC No: 25100 standard [4]. This standard includes the parameters that must be provided at the end of the beamline, and these parameters are shown in TABLE I.

TABLE I. The requirements of ESA/ESCC No: 25100 Standard for SEE tests.

\begin{tabular}{|c|c|}
\hline Parameters & Value \\
\hline Energy range & $20 \mathrm{MeV}-200 \mathrm{MeV}$ \\
\hline Flux range & $10^{5} \mathrm{p} / \mathrm{cm}^{2} / \mathrm{s}$ to at least $10^{8} \mathrm{p} / \mathrm{cm}^{2} / \mathrm{s}$ \\
\hline Beam area & $15.40 \times 21.55 \mathrm{~cm}$ \\
\hline Uniformity & $\pm 10 \%$ \\
\hline
\end{tabular}

The 2D design of the METU-DBL is shown in Fig. 2. There is a 5-port switching magnet at the beginning of the beamline, which can guide the beam into 5 different arms to perform different experiments.

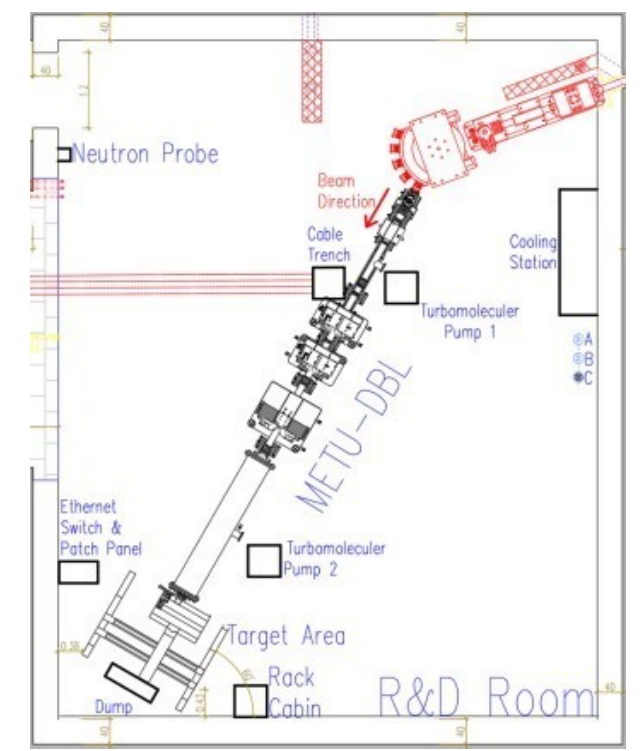

Fig. 2. A 2D drawing of METU-DBL and the R\&D room. A 5-port switching magnet is shown in red color at the beginning point of METU-DBL. The length of the beamline is approximately $7.5 \mathrm{~m}$ starting from the 5-port switching magnet.
The METU-DBL is installed on the right-most port of the switching magnet.

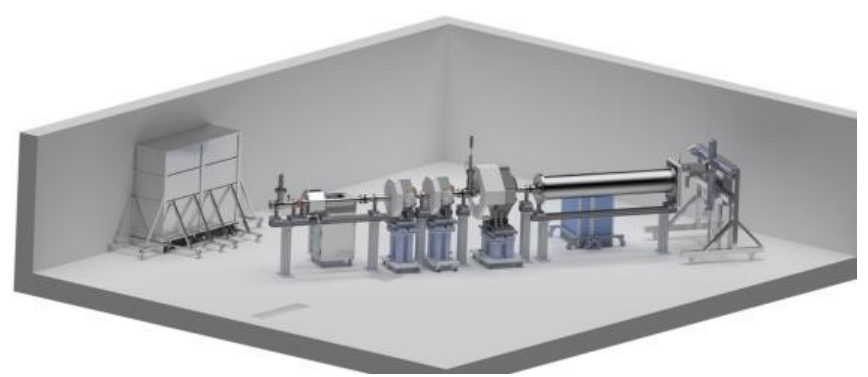

Fig. 3. Final design of METU-DBL. The beamline starts at the exit of the 5port magnet. A large custom-design quadrupole magnet follows two smaller ones. The beam pipe gets wider as the beam blows up towards the end of the beamline. 5-port switching magnet and radiation shields are not shown.

The final setup of METU-DBL includes a beam stopper and a vacuum shutter to protect the beamline and the vacuum of METU-DBL. A helium section improves the uniformity at the target area by scattering the beam between the titanium foils. An adjustable conical collimator is used to provide the desired flux and also to protect the magnets of METU-DBL. 3 quadrupole magnets is employed to enlarge the beam. A beam measurement system and a beam dump towards the end of the beamline are shown in Fig. 3 [5].

METU-DBL provides users a wide flux range to choose from $\left(10^{5}-10^{11} \mathrm{p} / \mathrm{cm}^{2} / \mathrm{s}\right)$ and an energy range of $15-$ $30 \mathrm{MeV}$. Reaching fluences of $10^{14} \mathrm{p} / \mathrm{cm}^{2}$ only takes about 20 minutes.

METU-DBL has six subsystems: beam optics, vacuum, cooling, robotic, control, test and measurement subsystems.

Beam optics studies of METU-DBL follow the ESA/ESCC No. 25100 standard. This subsystem consists of quadrupole magnets to enlarge the proton beam, collimators to reduce the proton flux and a beam screen to observe the beam profile. $\mathrm{C}++$ based MAD-X [6] and FORTRAN based TRANSPORT were used for the beam optics calculations [7]. A movable Aluminum Oxide screen, at $45^{\circ}$ to the beamline and ImageJ program [8] is used to analyze the beam profile.

In METU-DBL, protons must reach the target area with minimum energy loss to achieve the ESA/ESCC No: 25100 standard. A vacuum subsystem consisting of one mechanical pump and two turbomolecular pumps decreases the pressure inside the beam pipe to $10^{-6} \mathrm{mbar}$ is which is the desired vacuum level.

Quadrupole magnets and collimators of METU- DBL get warm due to high currents and secondary particles during irradiation tests. The cooling subsystem of METUDBL consists of a water tank and 2 circulation pumps providing $50 \mathrm{~kW}$ of cooling power. The water in the tank is sent to the beam elements and quadrupole magnets by circulation pumps. Also, thermal analyses of the beam elements directly exposed to the proton beam are simulated using ANSYS Fluent [9] to make sure no element will be deformed by thermal loads. An overhead of $15 \mathrm{~kW}$ is included in the cooling budget for the cooling requirements of future users of METU- DBL. 
The motions of the electromechanical components are controlled by the METU-DBL robotic subsystem. This subsystem is in charge of the management of the test table components (detectors as well as the devices under tests), the control of the adjustable conical collimator and a mobile radiation measurement robot. These tasks are performed either autonomously or by user commands.

METU-DBL control subsystem provides the communication and the control of the electronics and electromechanics. Control subsystem consists of control software, an industrial computer, a control computer, PLCs, motor drivers and Ethernet switches. During radiation tests, each component of the beamline is monitored from the TAEA SANAEM PAF control room.

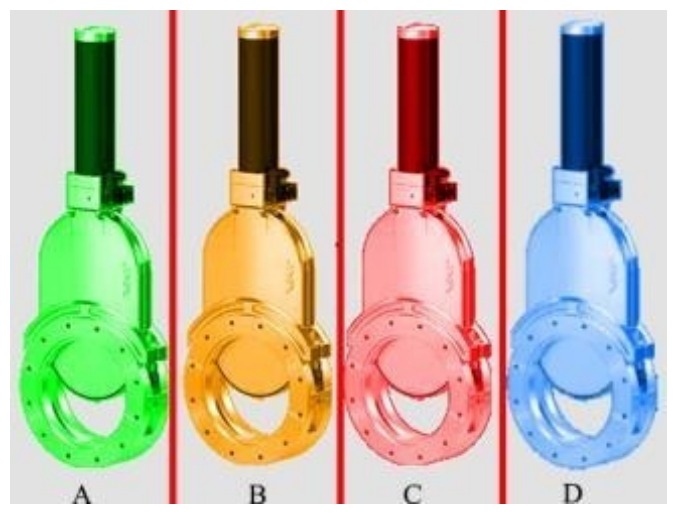

Fig. 4. Color codes of the beam elements of METU-DBL. While green color shows the ideal status, yellow color indicates the warning status. The red color shows the error status and the blue color indicates the safe status.

In the Fig. 4, the instant states of each beam element are indicated by color codes. Green is an ideal condition; yellow in case of a warning, red in case of error, and the blue is the safe condition. This visual aid allows for a fast intervention in case of an emergency.

The test and measurement system has three different detectors: TimePix 3 pixel, fiber scintillators and diamond detector which are shown in Fig. 5. These are necessary to measure the proton beam flux and the uniformity of METU-DBL target area.

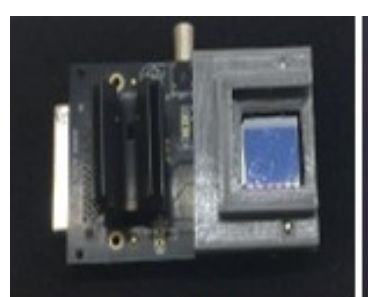

(a)

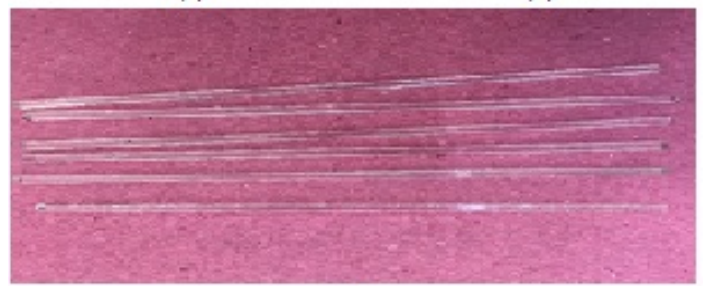

(c)

Fig. 5. Detectors of METU-DBL (a) Timepix3 pixel detector, (b) diamond detector (c) fiber scintillator.

The Timepix 3 is a pixel detector which was developed within the Medipix collaboration at CERN. The critical part of this detector is the sensor layer, and it is segmented with a pixel size of $55 \mu \mathrm{m}$ into a square matrix of $256 \mathrm{x}$ 256 pixels [10]. These pixels have a threshold for particle measurement, and they provide information about particles having more energy than the pre-defined threshold energy. Proton flux may also be calculated with fiber scintillators. The number of photons that are created is proportional to the number of protons reaching the scintillator. The proton flux can be inferred by measuring the photon flux in the fiber scintillator [11]. Due to its radiation endurance, diamond detectors [12] can also be used to measure proton flux. These features enable them to work efficiently for a long time under irradiation. Other detectors' performance might suffer because of radiation damage sustained to their crystal structure. The detector has a single diamond crystal between two gold electrodes.

When a proton passes the crystal, a potential difference occurs between the electrodes that can be used for proton detection. Under this proton irradiation, activation of the materials that exist around the beamline might occur. In high radiation environment dose calculations (for example Hi Lumi LHC), radioisotope and shielding studies are important to protect the beam elements, the sensitive electronic devices that are exposed to radiation directly or indirectly, and the health of the radiation workers. In METU-DBL, the instantaneous dose rate in the R\&D room is recorded with Geiger-Müller, gamma and neutron detectors. During radiation tests, the maximum dose rates and cooling times are also recorded.

In addition, in the scope of dose calculations and shielding studies, METU-DBL system was built into the FLUKA simulation program [13]. FLUKA is a tool for the calculations of particle transportation and interactions with matter. FLUKA packages include proton and electron accelerators, calorimetry, activation, dosimetry, detector design, accelerator driven systems, etc. The radioisotope and dose rates of the beam elements can be analyzed with FLUKA.

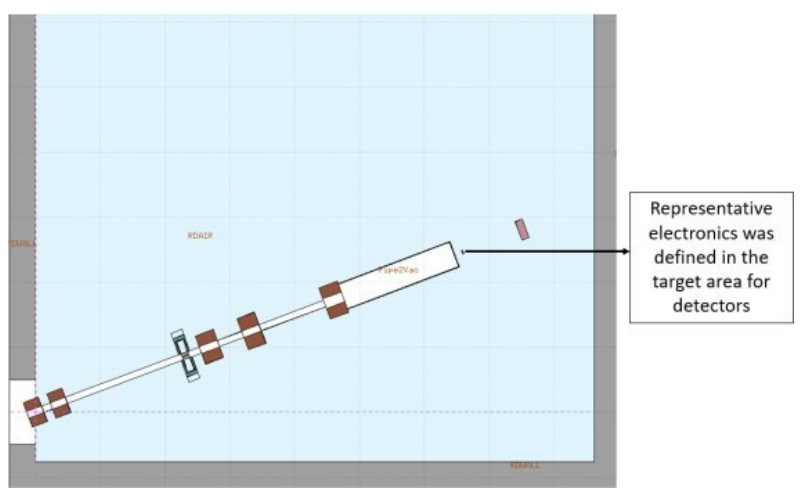

Fig. 6. Modeling of METU-DBL as seen in the graphical interface of FLUKA called Flair. Beam elements such as magnets are seen as brown rectangles around the beam pipe. An electronic component in the target area was placed to represent detectors and their readout electronics to determine the necessary shielding designs.

For the shielding designs, various material thicknesses and sequences were simulated. In order to stop protons, secondary neutrons, and gammas; aluminum, polyethylene, and lead were used respectively. Numerous designs were studied for shielding the detectors, and the best results depending on availability and manufacturability were chosen for each. The shielded 
TimePix 3 pixel detector, one of the fiber scintillators, and the diamond detector is shown in Fig. 7.

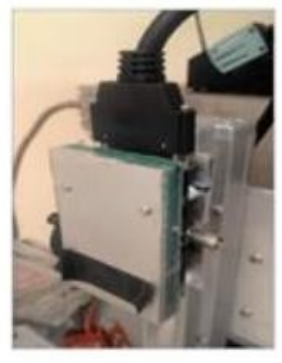

(a)

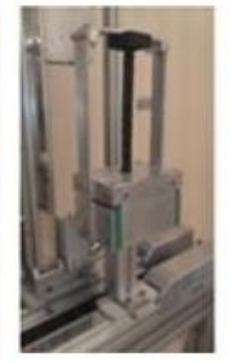

(b)

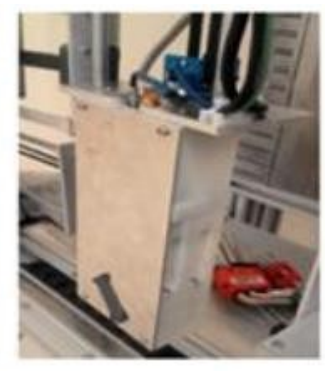

(c)
Fig. 7. From left to right, (a) the shielded TimePix 3 pixel detector, (b) one shielded fiber scintillator, and (c) the shielded diamond detector can be seen. Numerous designs were studied for these detectors and the best results were chosen for each.

In the next section, pretest of METU-DBL will be explained.

\section{PRETESTS OF METU-DBL}

METU-DBL installation was performed by considering the shielding studies and dose calculations. During the pretests, a lot of experience has been gained, and the final installation started with the experiences from the pretests. Radiation tests of nine electronic cards and materials of eight different institutions were tested with the pretest setup between January and March 2018 [14].

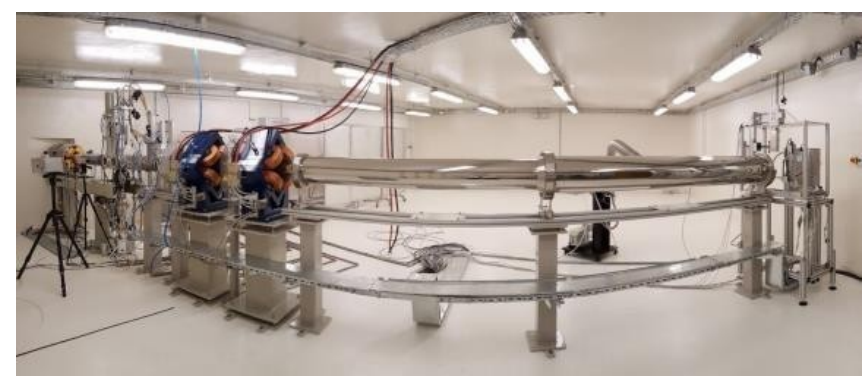

Fig. 8. Panoramic photo of the preliminary test design. The beam comes from the accelerator from beamline in the left wall. Initial beam diagnostics are made with CCD camera which looks at the image of the beam on an $\mathrm{Al}_{2} \mathrm{O}_{3}$ screen. The test and measurement system at the target area measures the flux and uniformity of the beam with 3 movable detectors. There is a beam dump before the beam hits the right wall. The total length of pretest line of METU- DBL is $6.97 \mathrm{~m}$.

In the pretest setup, the beam size could be as large as $4 \mathrm{~cm} \times 6 \mathrm{~cm}$ and the flux was between $1 \times 10^{8}$ and $1 \times 10^{10}$ protons $/ \mathrm{cm}^{2} / \mathrm{s}$. The preliminary setup of the METU-DBL at TAEA R\&D room is seen in the photograph shown in Fig. 8. The beam comes from the accelerator to beamline connection situated on the left wall. Initial beam diagnostics are made with a CCD camera which looks at the image of the beam on an $\mathrm{Al}_{2} \mathrm{O}_{3}$ screen. The test and measurement system at the target area measures the flux and uniformity of the beam using the diamond detector, TimePix 3 pixel detector and the fiber scintillator, all shielded and positioned on a movable setup. After passing through the measurement system, the beam encounters a beam dump at the right wall to stop the protons. The total length of METU-DBL pretest setup is $6.97 \mathrm{~m}$.

During the pretests stage, the performed radiation tests are shown in TABLE II. Each test was performed using $30 \mathrm{MeV}$ energy protons at different irradiation times. Only the pin diode test was performed at $2.0 \mu \mathrm{A}$, while the other tests were performed at $0.1 \mu \mathrm{A}$ as specified by the users.

TABLE II. Radiation tests which performed during the pretest of METUDBL.

\begin{tabular}{|c|c|c|}
\hline Institution & Material Type & Irradiation Time \\
\hline $\begin{array}{l}\text { METU- Department of } \\
\text { Physics }\end{array}$ & PIN Diode & $\begin{array}{l}30 \mathrm{~min} . \\
51 \mathrm{sec} .\end{array}$ \\
\hline $\begin{array}{l}\text { TÜBİTAK MAM } \\
\text { Materials Institute }\end{array}$ & $\begin{array}{l}\text { Solar cells for } \\
\text { IMECE Satellite }\end{array}$ & $\begin{array}{l}20 \mathrm{~min} . \\
1 \mathrm{sec} .\end{array}$ \\
\hline $\begin{array}{l}\text { TÜBİTAK MAM } \\
\text { Materials Institute }\end{array}$ & $\begin{array}{l}\text { Shielding glass } \\
\text { for IMECE } \\
\text { Satellite }\end{array}$ & $\begin{array}{l}49 \mathrm{~min} . \\
56 \mathrm{sec} .\end{array}$ \\
\hline $\begin{array}{l}\text { TÜBİTAK MAM } \\
\text { Energy Institute }\end{array}$ & $\begin{array}{l}\text { Battery } \\
\text { materials for } \\
\text { IMECE Satellite }\end{array}$ & $25 \mathrm{sec}$. \\
\hline Gazi University & Solar cells & $\begin{array}{l}9 \mathrm{~min} . \\
57 \mathrm{sec} .\end{array}$ \\
\hline TÜBİTAK Uzay & GaN-FET & $\begin{array}{l}30 \mathrm{~min} . \\
42 \mathrm{sec} .\end{array}$ \\
\hline Sabancı University & $\begin{array}{l}\text { Coating } \\
\text { substance }\end{array}$ & $55 \mathrm{sec}$. \\
\hline $\begin{array}{l}\text { METU- Department of } \\
\text { Metallurgical and } \\
\text { Materials Engineering }\end{array}$ & $\begin{array}{l}\text { Bulk metallic } \\
\text { glass }\end{array}$ & $30 \mathrm{~min}$ \\
\hline $\begin{array}{l}\text { TÜBİTAK Bं̈LGEM } \\
\text { UEKAE YITAL }\end{array}$ & DDA3 & $10 \mathrm{~min}$. \\
\hline
\end{tabular}

During the Pin Diode radiation tests, the arrangement of the 3 detectors on the test table with pretest setup is shown in Fig. 9. The TimePix3 pixel detector and the fiber scintillators were attached to pin diodes for readout and a diamond detector. This system has movable X-Y stages onto which the detectors are attached. The target area is scanned with $3 \mathrm{~mm}$ accuracy and flux measurement are taken at regular intervals. The device under test is positioned based on these measurements so that the required homogeneity is met.

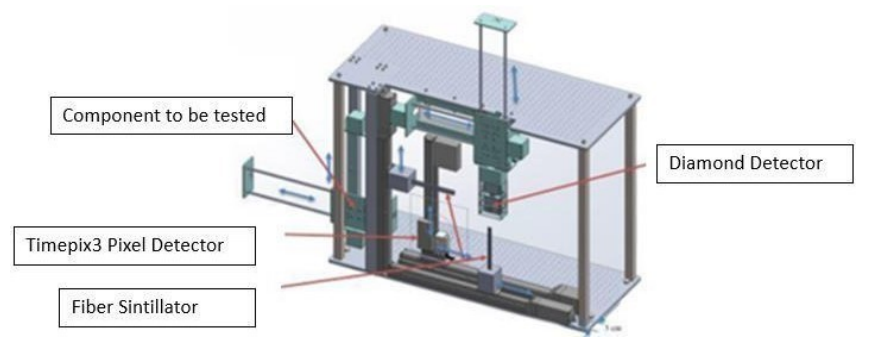

Fig. 9. Pretest test table of METU-DBL. Three detectors which are TimePix3 pixel detector, fiber scintillators are seen. The target area is scanned with 3 $\mathrm{mm}$ accuracy and flux measurement are taken at regular intervals. Device under test is positioned based on these measurements so that the required homogeneity is met.

Before the tests, detectors take measurement to ascertain the integrity of the beam at the target area. The components to be tested then are placed on the scanned area with detectors. First proton irradiation test was performed on six pin-diodes. The position of the pin diodes on the test table (left) and measurement positions and fluxes for each measurement taken by the diamond detector (right) are shown Fig. 10. The maximum flux $\left(1.88 \times 10^{11} \mathrm{p} / \mathrm{cm}^{2} / \mathrm{s}\right)$ and minimum flux $\left(4.82 \times 10^{10}\right.$ $\mathrm{p} / \mathrm{cm}^{2} / \mathrm{s}$ ) are seen in the test area (red rectangular). Characterization of the pin diodes before and after testing have been performed and are being prepared for publication. 

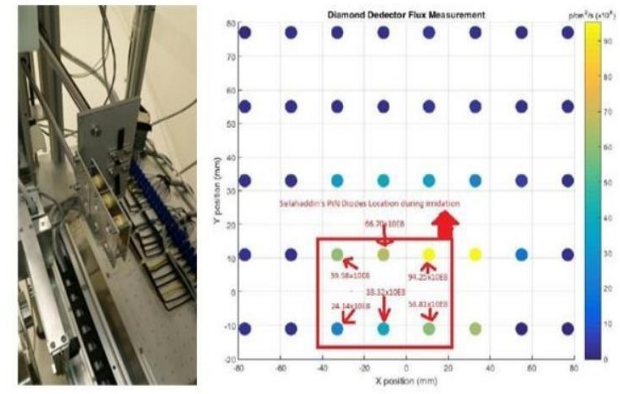

Fig. 10. In (a), the pin diodes under test are seen in the movable holder of the test desk. In (b), measurement positions and fluxes for each measurement taken by the diamond detector (right) is shown with a color scale. The maximum flux $\left(1.88 \times 10^{11} \mathrm{p} / \mathrm{cm}^{2} / \mathrm{s}\right)$ and minimum flux $\left(4.82 \times 10^{10} \mathrm{p} / \mathrm{cm}^{2} / \mathrm{s}\right)$ seen in the test area shown in red.

After the pretests had been completed, the pretest line was disassembled, and the assembly of the beamline started according to the final design of METU-DBL.

\section{INSTALLATION OF THE FINAL SETUP OF METU- DBL}

The main differences between the beamline setup in the pretests stage and the final configuration are the employing of a 5-port switching magnet, movable beam screen, custom design quadrupole magnet, new conical collimator and helium section. The differences are summarized in TABLE III.

TABLE III. Differences between the pretest and final design of METUDBL.

\begin{tabular}{|l|l|}
\hline \multicolumn{1}{|c|}{ Pretest Line Design } & \multicolumn{1}{c|}{ Final Design of METU-DBL } \\
\hline \multicolumn{1}{|c|}{-} & 5-Port Switching Magnet \\
\hline 2 Quadrupole Magnets & $\begin{array}{l}\text { 2 Quadrupole Magnets and Custom } \\
\text { Design Quadrupole Magnet }\end{array}$ \\
\hline Conical Collimator & New Conical Collimator \\
\hline Fixed Beam Screen & Movable Beam Screen \\
\hline \multicolumn{1}{c|}{-} & Helium Chamber \\
\hline Test Table & New Test Table \\
\hline
\end{tabular}

As of May 2019, production and assembly of 5-port switching magnet, custom design quadrupole magnet, and new test mechanism have been completed and added to the beamline. The helium section, movable beam screen and new conical collimator are in the design and production stages.

The final setup installation started with the assembly of a 3 tons 5-port switching magnet, and it is located at the starting point of the beamline as shown in Fig. 11. This magnet was purchased from IBA and mounting process were performed with the IBA technical team. The METUDBL installation started on the right-most port of the switching magnet.

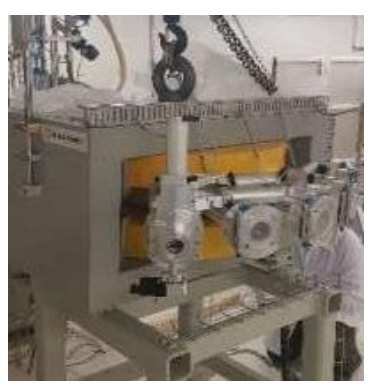

Fig. 11. 5-port magnet installed in the R\&D room of the TAEA SANAEM PAF. METU-DBL beamline follows from the right- most exit of this magnet.
The other critical elements of beam optics are three quadrupole magnets in the METU-DBL which are shown in Fig. 12. Quadrupole magnets focus the beam in one direction, while defocusing in the other direction. The incoming proton beam is enlarged by quadrupole magnets to satisfy the ESA/ESCC No: 25100 standard.

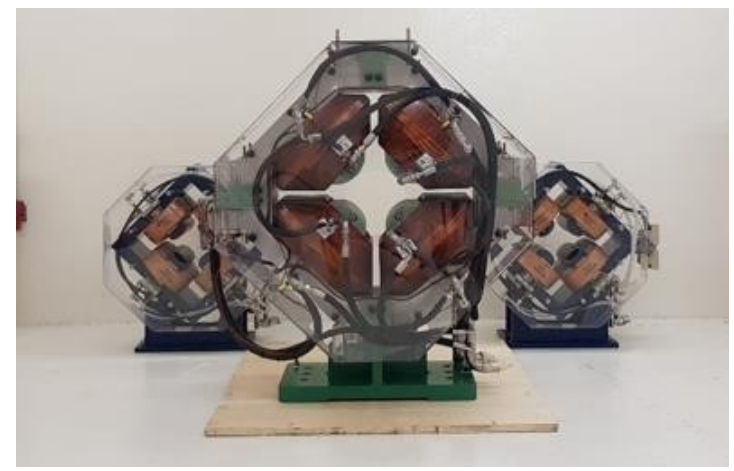

Fig. 12. Quadrupole magnets of METU-DBL. Custom design quadrupole magnet can be seen at front and first two quadrupole magnets which purchased from Scanditronix can be seen at behind the custom design magnet.

The first two quadrupole magnets of METU-DBL were purchased from Scanditronix Company, and the third quadrupole magnet was designed by the METU-DBL team and manufactured in Turkey, since it was not produced commercially.

METU-DBL pretests were performed with only the first two quadrupole magnets. The properties of these quadrupole magnets in comparison to the new custom design quadrupole magnet are given in the TABLE IV.

TABLE IV. Properties of Quadrupole Magnets of METU-DBL.

\begin{tabular}{|l|c|c|}
\hline & $\begin{array}{c}\text { First Two } \\
\text { Quadrupole } \\
\text { Magnets }\end{array}$ & $\begin{array}{c}\text { Custom Design } \\
\text { Quadrupole } \\
\text { Magnet }\end{array}$ \\
\hline Flow Rate & $3.31 / \mathrm{min}$ & $3.41 / \mathrm{min}$ \\
\hline Current & $70 \mathrm{~A}(\mathrm{nom})$. & $160 \mathrm{~A}$ \\
\hline Aperture & $110 \mathrm{~mm}$ & $160 \mathrm{~mm}$ \\
\hline Gradient Strength & $5.0 \mathrm{~T} / \mathrm{m}$ & $7.5 \mathrm{~T} / \mathrm{m}$ \\
\hline Effective Length & $300 \mathrm{~mm}$ & $300 \mathrm{~mm}$ \\
\hline Number of turns per coils & 86 & 122 \\
\hline
\end{tabular}

In TABLE IV, gradient strength is the most critical parameter for quadrupole magnets. Because ESA/ESCC No: 25100 standard can only be achieved with the given gradient strength values.

Electrical and mechanical acceptance tests of first and second quadrupole magnets were performed at METU Physics Department and the magnetic field measurements were made by TUBITAK UME. The custom design quadrupole magnet underwent mechanical, electronic and magnetic tests, first in Turkey, and then in CERN. The magnet has successfully passed all acceptance tests and was therefore certified by CERN.

In accelerator systems, another essential beam elements are collimators to cut the tails of the beam. Thus, the size of the beam in the $x-y$ axes is reduced, and the number of particles is decreased.

The function of the conical collimator in METU- DBL is to reduce the flux. After the pretests, a collimator of 40 $\mathrm{x} 40 \times 28 \mathrm{~cm}^{3}$ dimensions with an aperture of $1 \times 1 \mathrm{~mm}^{2}$ to $10 \times 10 \mathrm{~mm}^{2}$ was designed to precisely center the gap 
where the proton beam passes and to provide sufficient cooling. Different flux options can be offered to users in the target area, with the adjustable aperture of the conical collimator. The design of the conical collimator is given in Fig. 13.

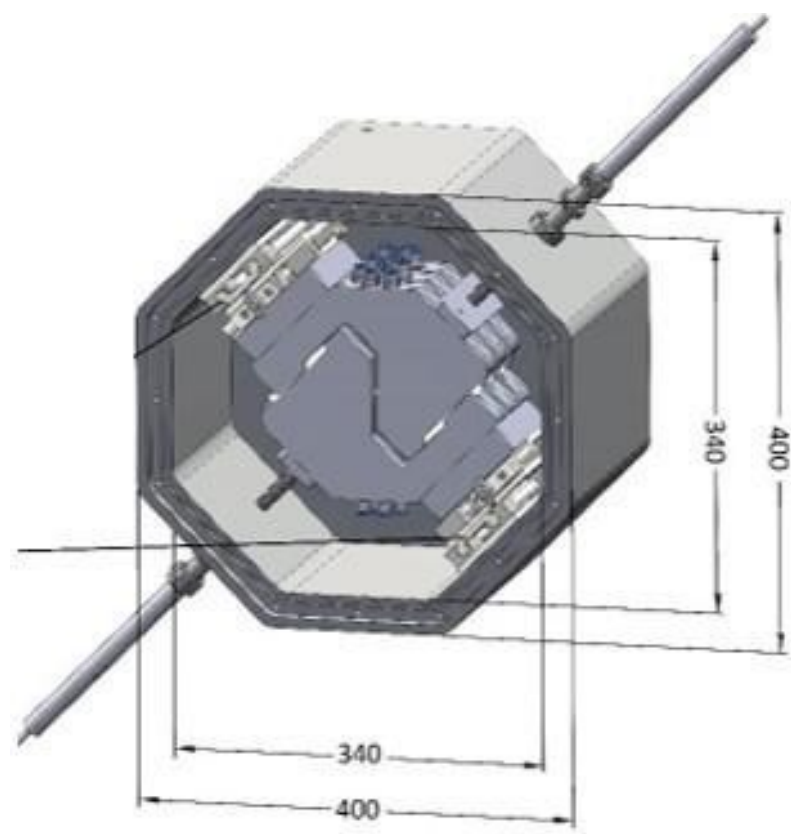

Fig. 13. Conical Collimator design of METU-DBL. Linear drives control leaves which cut the incoming beam. (All dimensions are given in millimeters).

During the pretests, mounting and dismantling the fixed beam screen into the beamline was time consuming. A movable beam screen was designed to increase the efficiency of the final setup of METU-DBL.

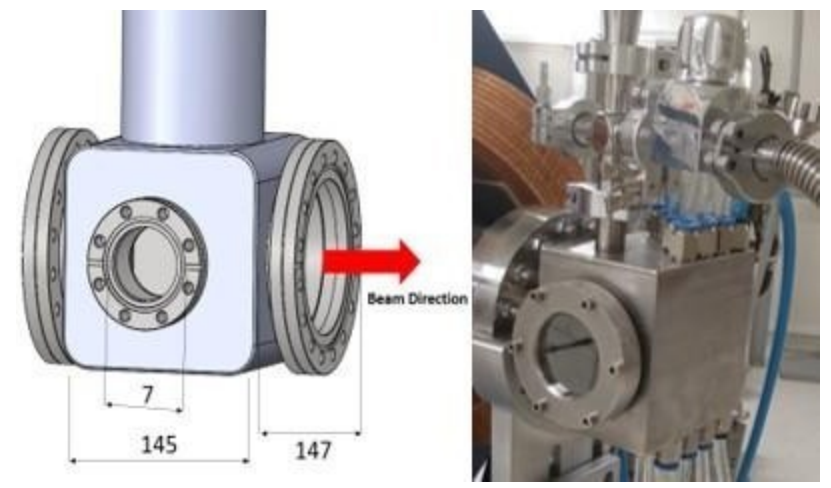

Fig. 14. A 3-D drawing of the movable beam screen designed to observe the proton beam profile. The image of the beam can be viewed from the window which has $7 \mathrm{~cm}$ radius (Left). Fixed Beam screen of METU-DBL which is used for pretest. Also the radius of fixed beam screen is $7 \mathrm{~cm}$. (All dimensions are given in millimeters).

On the movable beam screen, an $\mathrm{Al}_{2} \mathrm{O}_{3}$ plate of $7 \times 7$ $\mathrm{cm}^{2}$ size was placed at an angle of $45^{\circ}$ to the beam axis and was used to find the center of the beam. Also, sapphire glass was preferred for the glass of the moving beam screen due to its radiation endurance. The setup is shown in Fig. 14. This screen is essential for alignment of the mechanical installation of the accelerators to observe the beam profile at different stages. In the METU-DBL pretest setup, the size of the proton beam can be measured as a function of a magnetic field by using a quadrupole scan technique [15] with a fixed beam screen. The quadrupole scan technique is a widely used method for measuring transverse emittance at particle accelerators.

In METU-DBL, two scattering foils of $50 \mu \mathrm{m}$ thickness provide a homogeneous radiation field in the target area by enlarging the beam and reducing the proton flux. When the particles pass through the scattering foils, the angle of particles change due to multiple Coulomb scattering. The protons lose their energy while passing through scattering foils, heating the foils up in the process, thus cooling is required. The protons at a current of $100 \mu \mathrm{A}$ of $30 \mathrm{MeV}$ have a thermal load of $3 \mathrm{~kW}$. A helium section which has $150 \times 100 \times 140 \mathrm{~mm}^{3}$ dimensions is placed between the scattering foils to provide the cooling requirements. The cross-sectional area of the helium cooling section is shown in Fig. 15. In this design, stainless steel 316 was chosen as the material of the helium section.

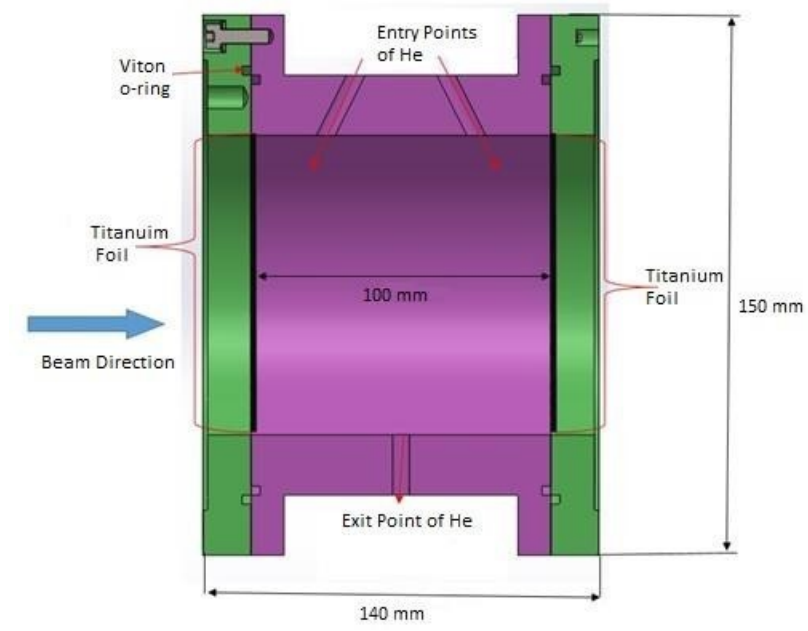

Fig. 15. Helium cooling chamber design of METU-DBL. The titanium foils were placed between the flanges shown in green and purple colors.

In this design, the titanium foils were placed in contact with the surface of the viton o-rings between the flanges shown in green and purple colors. It is aimed to provide more efficient cooling for titanium foils by giving $45^{\circ}$ angles to helium gas ducts. The helium gas entering the chamber with two channels is intended to exit from a single point and go to the heat exchanger that cools the helium gas.

The last improvement in METU-DBL is the new test table. The first design of the test table with detectors and DUTs was used throughout the pretests. During the pretest, the risk of collision of detectors with each other was high due to the small size of the previous test table. After the completion of the pretests, the test table was redesigned in the light of the acquired experience, and the final design was completed. The previous test table could scan the area which has limit of $\pm 300 \mathrm{~mm}$ in $\mathrm{X}$-axis and $\pm 1200 \mathrm{~mm}$ in $\mathrm{Y}$-axis while the new test system could scan the larger area, which has the limit of $\pm 760 \mathrm{~mm}$ in $\mathrm{X}$-axis and $\pm 670 \mathrm{~mm}$ in Y-axis. It can be seen in Fig. 17 . 


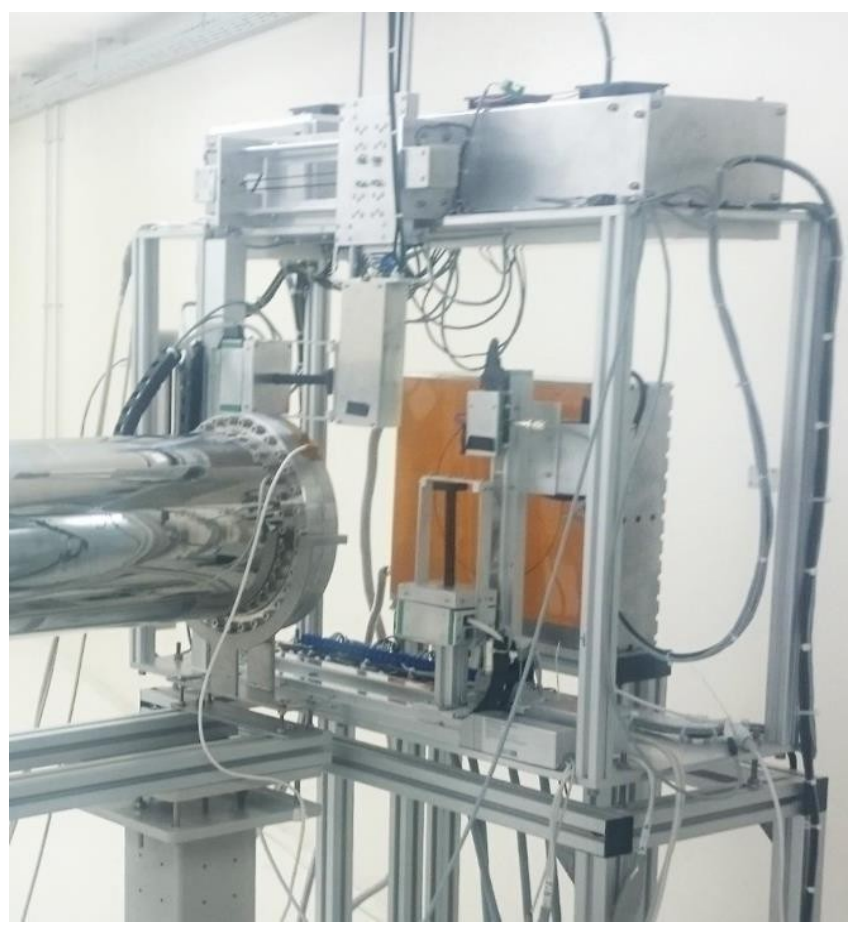

Fig. 16. Test table of METU-DBL pretest.

The detectors and DUTs are attached in the crossshaped holders and are moved by the servo motors in the new design of METU-DBL test mechanism. The speed, acceleration and position control devices of the servo motors can be set independently of the PLC or can be controlled manually using a software.

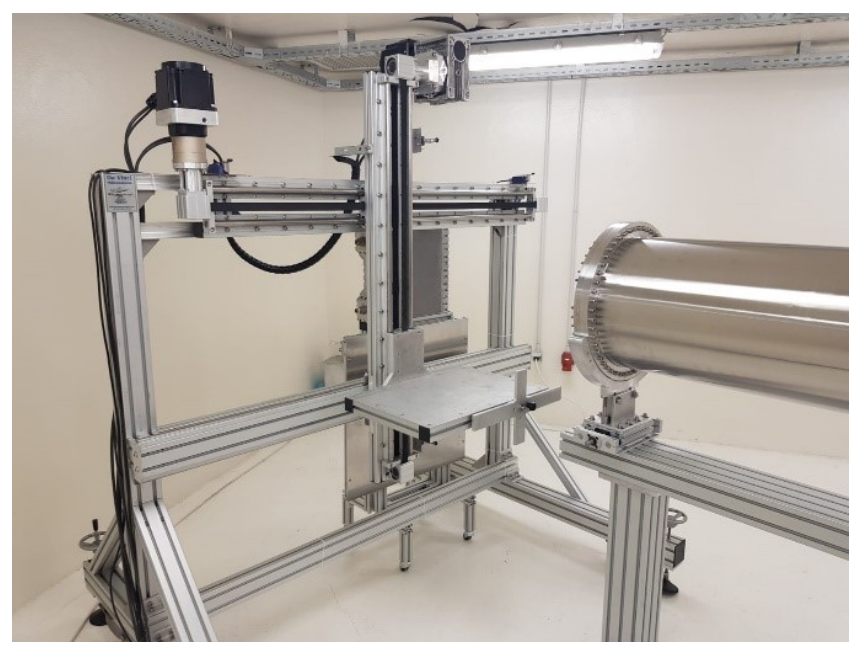

Fig. 17. Final design of the test table of METU-DBL, followed by the beam dump.

\section{CONCLUSION}

METU-DBL pretest installation was completed, and nine radiation tests were performed between January and March 2018. After the pretests, the METU-DBL system was dismantled and re-established in accordance with the final design. In contrast to the pretest system, 5-portswitching magnet, custom design quadrupole magnet and helium chamber were added to the beamline. Also, the design of the conical collimator and beam screen has been updated. Radiation tests will be carried out when the METU-DBL installation is completed. Commissioning and tests of electronics will commence in 2019. METU-DBL will serve for testing components intended for high radiation environments such as the Hi Lumi LHC as well as medical and space applications.

\section{ACKNOWLEDGMENT}

METU-DBL is funded by Strategy and Budget Presidency (Grant no: 2015K121190) and also supported by the Turkish Academy of Sciences. We want to thank our colleagues from TAEA SANAEM Proton Accelerator Facility.

\section{REFERENCES}

[1] Bruce, R., A. Lechner, and S. Redaelli. Energy deposition simulations for upgraded collimation layouts. No. CERN-ACC2014-0009. 2014

[2] Underwood, Craig I. The single-event-effect behaviour of commercial-off-the-shelf memory devices. A decade in low-Earth orbit. In: RADECS 97. Fourth European Conference on Radiation and its Effects on Components and Systems (Cat. No. 97TH8294). IEEE, 1997. p. 251-258.

[3] TAEA, "Proton Accelerator Facility Booklet", TAEA SANAEM, 2012

[4] ESA, "Single Event Effects Test Method and Guidelines, ESCC Basic Specification No. 25100", 2014

[5] Demirkoz, M. Bilge, et al. Pretest setup installation of the METUDBL project to perform space radiation tests. In: 2017 8th International Conference on RAST 2017. IEEE, p. 311-316.

[6] Schmidt, F., \& Grote, H. (2005). MAD-X User's guide.

[7] Rohrer, U. (2007). PSI graphic transport framework. based on a CERN-SLAC-FERMILAB version by KL Brown et al.

[8] Abràmoff, M. D., Magalhães, P. J., \& Ram, S. J. (2004). Image processing with ImageJ. Biophotonics international, 11(7), 36-42.

[9] Fluent, A. N. S. Y. S. "Ansys fluent." Academic Research. Release 14 (2015).

[10] Kraus, V., Holik, M., Jakubek, J., Kroupa, M., Soukup, P., \& Vykydal, Z. (2011). FITPix-fast interface for Timepix pixel detectors. Journal of Instrumentation, 6(01), C01079.

[11] Beischer, B., Gast, H., Greim, R., Karpinski, W., Kirn, T., Nakada, T., \& Wlochal, M. (2010). A high-resolution scintillating fiber tracker with silicon photomultiplier array readout. Nuclear Instruments and Methods in Physics Research Section A: Accelerators, Spectrometers, Detectors and Associated Equipment, 622(3), 542-554.

[12] Rebai, M., Cazzaniga, C., Croci, G., Tardocchi, M., Cippo, E. P., Calvani, P., \& Gorini, G. (2015). Pixelated Single- crystal Diamond Detector for fast neutron measurements. Journal of Instrumentation, 10(03), C03016.

[13] Battistoni, Giuseppe, et al. (2015) Overview of the FLUKA code, Annals of Nuclear Energy 82, p: 10-18.

[14] Demirkoz, B., Nigdelioglu, S., Yigitoglu, M., Aydın, S., Efthymiopoulos, I. (2018). METU defocusing beamline project for the first SEE tests in Turkey and the test results from the METUDBL preliminary setup. Nucl. Instrum. Meth. A., in press

[15] Green, A., \& Shin, Y. M. (2015). Implementation of Quadrupolescan Emittance Measurement at Fermilab's Advanced Superconducting Test Accelerator(ASTA) 